\title{
GRAVITONS IN DE SITTER SPACE
}

\author{
Bruce ALLEN \\ Department of Physics and Astronomy \\ Tufts University \\ Medford, MA 02155 \\ U.S.A.
}

Everyone knows that "Einstein's greatest mistake", the cosmological constant $\Lambda$, is very close to zero $|1|$. There have been many attempts to explain why $\Lambda$ must be exactly zero $|2|$, but none of these efforts has succeeded. In fact it is now fashionable to believe that during the very early history of the universe the value of $\Lambda$ was quite large $|3|$. This so-called "inflationary" epoch would have been a long period of exponentially rapid expansion, and would elegantly explain two otherwise mysterious observational truths : the universe is uncannily flat, and the cosmic microwave background radiation has no right to be as isotropic as it is.

There are three things that we would like to know. First, why is $\Lambda$ zero today? Second, could $\Lambda$ have been very big in the past ? And finally, if $\Lambda$ was very big in the past, what consequences would that have today ? Unfortunately this paper will not answer any of these questions, but I hope that it will nevertheless accomplish something useful. I am going to show that one of the answers that has recently been given to the first question above - Why is $\Lambda$ zero ? - is not correct. However, before I get into the technical nitty-gritty, let me give you a synopsis of the kinds of answers that have been suggested to these questions.

One answer which has been given to the question - why is $\Lambda$ equal to zero today?has been that zero is the only consistent answer. Let me reveal my predjudices at once and say that I don't believe this. First of all, it isn't borne out by careful calculation. For example, someone once decided that $\Lambda$ must be zero, because if it was not zero then a certain scattering amplitude would not be unitary. However a more careful investigation showed this to be false $|4|$. A different argument, which is being worked on by several people, is that if $\Lambda$ is not zero then particles get created out of the vacuum, and damp the value of $\Lambda$ to zero $|5|$.

The problem is this: What quantities to you calculate to see if $\Lambda$ really decays (or has to be zero from the outset) ? One may show, for example, that a scalar particle propagating in a background with $\Lambda$ nonzero will emit additional scalar particles, which will continue to do the same thing, and so on, ad infinitum. Now this sounds 
unstable. However if you calculate the energy-momentum tensor of this process, you find that it only shifts the value of $\Lambda$ a little bit $|6|$.

Another example : you can calculate the corrections to the stress-tensor due to the Plank-scale quantum fluctuations of the vacuum. Indeed, these corrections shift the value of $\Lambda$, and one can study the semi-classical back-reaction to find out what effect this has on the metric tensor. Do this, and you find that the metric is greatly altered. It sounds like a tremendous physical instability until you realize that the identical argument implies that flat space with $\Lambda=0$ can't be stable either $!|7|$. So here it is clear that something is wrong with the argument itself, since locally our spacetime is very flat, and shows no signs of decaying away beneath our feet !

One of the basic problems with these arguments is that the natural ground state (or vacuum state) for de Sitter space is time-reversal symmetric $|8|$. In this socalled Gibbons-Hawking vacuum state, it is impossible for particle creation to occur, because if the number of particles were increasing, that would break time-reversal symmetry.

If it could really be established that $\Lambda=0$ was the only consistent value, I don't think that it would be a good thing. The cosmological constant is a measure of the local energy density of the empty vacuum state. If it was truly zero then there would be no way to generate cosmological inflation, which would be very unfortunate. (This is another reason why I am inclined to believe that there is nothing which is inconsistent about $\Lambda$ nonzero). Because $\Lambda$ is simply a measure of the vacuum or latent energy, it can change during phase transitions, and it seems certain that if symmetry in gauge theories is restored at high temperatures then such phase transitions must have taken place, as our universe cooled and expanded $|9|$. So it seems quite possible that $\Lambda$ was nonzero in the past-and this leads to our final question above.

What kinds of effects would be associated with a large positive $\Lambda$ ? Well, first there would be classical "gravitational" effects. Separated particles, freely falling, would accelerate away from one another. At a certain distance the recessional velocity would become unity, and there would be a cosmological particle horizon $|10|$. A given observer could not see farther than this distance. In addition to these classical effects there would be quantum effects. The best known of these is the GibbonsHawking effect -a freely falling observer would see a thermal spectrum at temperature $\left(12 \pi^{2}\right)^{-1 / 2} \Lambda^{1 / 2}$ radiating from the imaginary surface which we have just describedthe observer's particle horizon $|8|$. There are probably other interesting effects too, but we don't know what they are yet. The subject of quantum field theory in de Sitter space is still in its infancy. In fact the only real results are that we know how to construct the Fock space of states, and how to find the correlation functions for spin $0, \frac{1}{2}$ and $1|11|$. We also know a 1ittle bit about interacting fields in de sitter space $|12|$. 
Now let me tell you the idea which I intend to spend the rest of this talk trying to destroy. The idea was to show that $\Lambda$ must be zero because of properties of gravitons when $\Lambda$ is nonzero. If we were trying to prove that $\Lambda=0$, then, in the absence of any exact symmetry or invariance which would force $\Lambda$ to vanish, this would be the next best thing. The reason is this : $\Lambda$ is only observable through relativity, because it represents an otherwise completely arbitrary zero-point for measuring the energy-density of space-time. Were it not for general relativity, or gravity, then any background energy density would be completely unobservable, and it could be set to zero with the stroke of a pen. However in the presence of gravity, the vacuumenergy density $\Lambda$ does become observable, for example through the classical effects described above. It would therefore be nice if the only thing (gravity) that enables us to observe $\Lambda$ in the first place would also carry with it some quantization consistency condition that would force $\Lambda$ to be zero. This would be an elegant solution to our problem : if gravity, the only force that allows us to observe $\Lambda$, demands that $\Lambda$ vanish for reasons of consistency.

An argument of this type has recently been made by Antoniadis, Iliopoulos and Tomaras $|13|$. They claim that if one quantizes gravitational fluctuations in the presence of a background energy density $\Lambda$, then the resulting theory shows a particular kind of inconsistency called an "infra-red divergence". Now these words can refer to any one of several different problems. For example saying that QED has infra-red divergences generally refers to the fact that external 1 ines in Feymman diagrams emit an infinite number of low-frequency photons |14|. However this is not a real problem because the energy carried away by this process is not infinite. Similarly, in the theory of massless scalar electro-dynamics, the effective action has an infra-red divergence, and a mass-scale must therefore be introduced into the theory. The gauge fields thus aquire a mass, and the gauge symmetry is broken $|15|$.

In the recent work by Antoniadis, Iliopoulos and Tomaras |13|, it was claimed that, because of an infra-red divergence, the two-point function of gravitational fiuctuations was infinite (regardless of the separation of the two points). They argued that this infinity caused certain tree-level scattering amplitudes to be infinite, and thus rendered the theory of quantum gravity inconsistent unless $\Lambda$ equaled zero.

What I am going to do in this talk is quite straightforward. First, I am going to talk about the graviton propagator, and explain why it is not, in and of itself, a physical object. In fact it depends upon the choice of gauge (by which, as I will shortly explain, I mean the choice of a gauge-fixing term). What this means in practice is that physical quantities (for example scattering amplitudes, or the expectation value of the curvature tensor) depend only upon certain components of the propagator. This dependence is just subtle enough so that the different graviton propagators, arising from different choices of gauge, give exactly the same physical result.

The next thing that $I$ will do is to show that it is indeed true that for certain choices of gauge, the graviton propagator is indeed infinite, exactly as claimed by Antoniadis et a1. However I am then going to show that there are other choices of gau- 
ge for which the propagator is completely finite! Then I will explain why this is so. The point will be that the gauge-fixing terms of Antoniadis et al. do not completely fix the gauge because they still allow a finite number of gauge transformations. It is for this reason that the propagator that they find is infinite. But this infinity is not a real physical divergence; it is an artifact of how the gauge-fixing was done. For a better choice of gauge, the propagator is completely finite! To make this point absolutely clear. I will then show that if one calculates scattering amplitudes in the Antoniadis et al. gauge, one still obtains a perfectly finite result, in spite of the fact that the graviton propagator is infinite. The reason for this is that the graviton propagator in their gauge is the sum of an infinite (unphysical gauge-artifact) term and a finite part. The infinite term does not contribute to the scattering amplitude of any interaction whose stress-tensor is conserved, and thus physical scattering amplitudes remain completely finite, contradicting the claims of Antoniadis et a). Although $I$ will not show it here, this cancellation takes place at higher orders as well. The point is that for good choices of gauge, the Fadeev-Popor ghosts are we11behaved; for a bad choice of gauge they introduce additional infra-red divergences in just the right way to cancel those arising from the gravitons.

So the real point of all this technical investigation is that, at least so far, there doesn't seem to be any intrinsic problem with $\Lambda \neq 0$. Of course it's entirely possible that something else will turn up in the future that will render de sitter space inconsistent; as things stand at the moment, it seems that we have to keep on thinking about it.

\section{Gauge-Fixing Terms and the Choice of Gauge.}

This is a straightforward subject, but one that a great many people seem to be unclear about. The source of most of the trouble is confusion about the relationship between the classical process called "choice of gauge" and its analogue in quantum field theory, which is called "choice of a gauge-fixing term" in the action. Let us begin by considering these two ideas, and the connections between them.

Suppose that $h_{a b}$ is a small perturbation of some background metric gab. Then there is a whole class of metric perturbations $h_{a b}$ that represent exactly the same physical perturbation. This is because under the infinitesimal coordinate transformation $x^{i} \rightarrow x^{i}+v^{i}$ the metric perturbation transforms into $\left.h_{a b}+\nabla_{(a} v_{b}\right)$. Since coordinate transformation does not cause any changes to physically observable quantities, we can conclude that the perturbations $h_{a b}$ and $\left.h_{a b}+\nabla_{(a} v_{b}\right)$ are gauge-equivalent. Any physical quantity, for example the perturbation of the curvature induced by $\left.h_{a b}+\nabla_{(a} V_{b}\right)$, will not depend upon $v_{b}|16|$. 
For this reason, in classical perturbation and stability theory, it is very common to "impose gauge conditions". The metric perturbations obviously lie in equivalence classes; two perturbations will be deemed equivalent iff they differ by $\nabla_{(a)} V_{b)}$ for some vector $V_{b}$. "Imposing gauge conditions" is a way to pick out one particular member from each equivalence class. For example one can impose the following conditions on $\mathrm{h}_{\mathrm{ab}}$,

$$
\begin{array}{rlrl}
\nabla^{a} h_{a b} & =0 & & \text { (transverse), } \\
h_{a}^{a}=0 & & \text { (traceless), } \\
t^{a^{a} h_{a b}}=0 & & \text { (synchronous), }
\end{array}
$$

to restrict the gauge freedom. Here $t^{a}$ is some arbitrary vector field (usually choosen to be timelike). These are not the only conditions that one could impose ; there are clearly an infinite number of other possibilities.

Now what about the quantum field theory of gravitational perturbations ? Well the action is a scalar and it is thus invariant under coordinate transformations, so the perturbations $h_{a b}$ and $h_{a b}+\nabla_{(a} V_{b}$ have exactly the same action; they are gauge equivalent. In this situation the standard thing to do is to add to the action an arbitrarily choosen term which is not invariant under the above transformation. This arbitrarily choosen gauge-fixing term breaks the gauge invariance. For example it could be,

$$
S_{\text {gauge }}=\int\left[\alpha\left(\nabla^{a_{h}}\right)^{2}+\beta\left(h_{a}^{a}\right)^{2}+\gamma\left(t^{a} h_{a b}\right)^{2}\right] d\left(v_{0} l\right),
$$

where at least one of the positive constants $(\alpha, \beta, \gamma)$ was nonzero. Now of course we have made a very arbitrary choice here; the Fadeev-Popov procedure allows us to compensate for this choice in just such a way that the scattering amplitudes are ultimately independent of it.

Now suppose that we have determined the euclidean propagator, which we could write for example as the path integral $|17|$

$$
G_{a b c^{\prime} d^{\prime}}\left(x, x^{\prime}\right)=\int d\left[h_{a b}\right] h_{a b}(x) h_{c^{\prime} d^{\prime}}\left(x^{\prime}\right) \exp \left(-s\left[h_{a b}\right]-S_{\text {gauge }}\left[h_{a b}\right]\right) .
$$

This propagator obviously depends upon the gauge-fixing parameters $\alpha, \beta$ and $\gamma$. Now suppose that we considered the divergence $\nabla^{a} G_{a b c} d^{\prime}$ as a function of $\alpha, \beta$ and $\gamma$. In general it would not vanish. However if $\alpha$ was taken to infinity, then it would vanish. The reason is that if $\alpha$ is very large then the field configurations in the action which don't have $\nabla_{\mathrm{a}} \mathrm{h}^{\mathrm{ab}}=0$ are exponentially suppressed by the gauge-fixing term. In the limit $\alpha \rightarrow \infty$ such configurations would make no contribution to the propagator. Similarly, if $\alpha, \beta$ and $\gamma$ were simultaneously taken to infinity, then the propagator would satisfy the "classical gauge conditions" (1.1) in that $\nabla^{a} G_{a b c} d^{\prime}$, 
$G^{a} a^{\prime} d^{\prime}$, and $t^{a} G_{a b c} d^{\prime}$ would all vanish. So we can see that the classical gauge conditions are obtained in quantum theory by singular choices of gauge. This is analogous to the situation in QED. There, if we wanted to have a transverse propagator satisfying $\nabla_{a}\left\langle A^{a} A^{b^{\prime}}\right\rangle=0$ we would need to choose Landau gauge ; ie use a gauge-fixing term $\lambda\left(\nabla_{\mu} A^{\mu}\right)^{2}$ in the action and take the limit $\lambda \rightarrow \infty$.

Now what gauge was used by Antoniadis et a1. $|13|$ ? In fact there were two possibilities that they considered. The first one was equivalent to the three conditions given before (1.1) where $t^{a}$ is a vector field orthogonal to a family of flat spatial surfacer which are the standard $k=0$ foliation of de Sitter space. In this choice of gauge the propagator for gravitons can be related to that of a pair of minimally coupled scalar fields in a simple manner. Indeed in this gauge (corresponding to taking $\alpha, \beta$ and $\gamma$ to infinity in (1.2)) the propagator is infinite. However it was not clear if the reason for this was because the three gauge-fixing parameters were becoming infinite, or if it was because the introduction of the vector field $t^{a}$ into the action was breaking de sitter invariance, or if it was because the gauge-conditions did not entirely determine the gauge. At that point in their work, Antoniadis et al. were not themselves certain if the infra-red divergence that they had discovered was a gauge artifact or not.

To resolve this uncertainty they then considered a second choice of gauge for which the gauge-fixing term was

$$
S_{\text {gauge }}=\lim _{\alpha \rightarrow \infty} \alpha \int\left[\nabla^{a}\left(h_{a b}-1 / 4 g_{a b} h_{c}^{c}\right)\right]^{2} d\left(V_{0} l\right) .
$$

In this case they also found an infra-red divergence in the propagator. They then carried out a tree-level scattering-amplitude calculation, and found an infinite result. This, they claimed, was proof that the infra-red divergence that they had found for two different gauge choices was truly a physical effect and not merely a gauge artifact.

We are going to concentrate on the second choice of gauge-fixing term (1.4) and will reach very different conclusions than those of Antoniadis et al. We are going to consider gauge-fixing terms of the following form, with $\alpha=1 / 2$,

$$
S_{\text {gauge }}=\alpha \int\left[\nabla^{a}\left(h_{a b}-\varepsilon g_{a b} h_{c}^{c}\right)\right]^{2} d\left(v_{0}\right)
$$

for all values of the constant $\varepsilon$. We are going to prove the following three statements :

1. The graviton propagator is finite if $\varepsilon$ does not equal one of the following "exceptional values" $(1 / 4,7 / 10,5 / 6, \ldots)$

$$
\varepsilon_{\text {exceptional }}=\left(n^{2}+3 n-3\right) /\left(n^{2}+3 n\right)
$$


for $n=1,2,3 \ldots$ If $\mathcal{E}$ has one of the exceptional values, then the propagator is infinite.

2. If $\mathcal{E}$ takes one of the exceptional values, then the propagator diverges because for that value of $\varepsilon$ the gauge-fixing term is not "sensitive" to a gauge transformation corresponding to a particular (finite set of) vectorfields $v^{a}$.

3. The scattering amplitude is finite and independent of the value of $\mathcal{E}$.

While the gauge fixing term that we consider has $\alpha=1 / 2$ and not $\alpha \rightarrow \infty$, our results apply equally well to the $\alpha=\infty$ gauge of Antoniadis et al. The reason why is because for the exceptional values of $\mathcal{E}$ the gauge-fixing-term fails to fix the gauge for any value of $\alpha$. In other words, when $\varepsilon$ takes one of the exceptional values (regardless of $\alpha$ ) then there exist certain perturbations $h_{a b}$ which are pure gauge $h_{a b}=\nabla_{(a} V_{b)} \neq 0$ and such that $S_{\text {gauge }}\left[\nabla_{(a} V_{b}\right]$ vanishes. Thus our conclusion will be that Antoniadis et a1. found an infra-red divergence only because they had the bad luck to choose an ineffective gauge-fixing term, and not because the graviton propagator in de Sitter space has any intrinsic physical infra-red divergence. For most choices of gauge the propagator would have been completely finite.

\section{How to find the Graviton Propagator.}

The basic idea of this section is to find a form for the graviton propagator which will make it easy for us to see how it depends upon the choice of a gauge-fixing term. For this purpose it turns out to be very convenient to represent the propagator as a mode sum. Such mode sums are very familiar in the context of Lorentzian space-time calculations of (for example) the commutator and symmetric functions for a scalar field. Here we are doing something slightly less familiar - a Euclidean mode sum. The point is this : in the Hawking-Gibbons vacuum state, which is de Sitter invariant, the two-point function only depends upon the geodesic distance between the two-points. It is also an analytic function for spacelike-separated points. Therefore if we can find this function for spacelike separations, its analytic continuation to timelike separation will yield all information about the physically interesting function, which is the Lorentzian two-point function.

Thus we will look for the two-point function only for spacelike separated points. One way to do this is to carry out the calculation on a Euclidean (++++) metric 4sphere of radius $a$, whose scalar curvature $R$ has the same constant value as the curvature of the physical Lorentzian de Sitter space $R=4 \Lambda$. On this four-sphere the distance between two points is always positive, so that spacelike separation is the only possibility. It can be easily shown $|18|$ that for such spacelike separation the two-point function on the sphere, considered just as a function of geodesic distance, 
is exactly the same as the Lorentzian two-point function for spatial separations. For that reason, from this moment on, we will perform all calculations on a foursphere of radius a and volume $8 \pi^{2} a^{4} / 3$. The cosmological constant is then $\Lambda=3 a^{-2}$.

To find the two-point function, we need to know the quadratic part of the gravitational action for a small metric fluctuation $h_{a b}$. This has been calculated in many places $|19|$. When we add to it the gauge-fixing term previously given (1.5), we find that the total gauged-fixed action is

$$
S+S_{\text {gauge }}=(64 \pi G)^{-1} \int h_{a b} w^{a b c d} h_{c d} d\left(V_{0}\right) .
$$

Here the second-order differential operator $w^{\text {abcd }}$ is given by

$$
\begin{aligned}
w_{a b c d}= & \left(\left[1-2 \varepsilon^{2}\right] g_{a b} g_{c d}-g_{c(a} g_{b}\right) d \\
& +(2 \varepsilon-1)\left(g_{a b} \nabla\left(c \nabla_{d}\right)+g_{c d} \nabla\left(2 g_{c(a} \nabla_{b}\right)\right.
\end{aligned}
$$

where $\mathcal{E}$ is the gauge-fixing parameter. Now the propagator is defined by the differential equation

$$
W_{a b c d} G^{c d a^{\prime} b^{\prime}}\left(X, X^{\prime}\right)=\delta_{\left(a^{a^{\prime}} \delta_{b)}^{b^{\prime}}\right.}
$$

together with the boundary conditions that $G^{\text {aba' }} b^{\prime}$ depend only upon the distance from $X$ to $X^{\prime}$ (in the sense of $|18|$ ) and that it only be singular if $X=X^{\prime}$.

This equation can be solved in many ways. One method that we have already exploited is to actually perform the path integral (1.3). This is done by choosing 10 "coordinates" in the space of all metric perturbations $h_{a b}$, and then integrating all of them from - $\infty$ to $\infty|20|$. A simpler method will be followed here; it involves using an ansatz which is justified by the previous method.

To use the simplest method, it is only necessary to have an orthogonal expansion of the delta function appearing on the right hand side of (2.3). This orthogonal expansion is

$$
\begin{aligned}
\delta_{\left(a^{2}\right.} \delta_{b} \delta^{b^{\prime}}= & \sum_{n=0}^{\infty} n_{a b}^{n}(x) h_{n}^{a^{\prime} b^{\prime}}\left(x^{\prime}\right)+\sum_{n=1}^{\infty} v_{a b}^{n}(x) v_{n}^{a^{\prime} b^{\prime}}\left(x^{\prime}\right)+\sum_{n=2}^{\infty} w_{a b}^{n}(x) w_{n}^{a^{\prime} b^{\prime}}\left(x^{\prime}\right) \\
& +\sum_{n=0}^{\infty} x_{a b}^{n}(x) x_{n}^{a^{\prime} b^{\prime}}\left(x^{\prime}\right) .
\end{aligned}
$$

Here the tensor fields $h_{n}^{a b}, v_{n}^{a b}, w_{n}^{a b}$ and $X_{n}^{a b}$ are all eigenfunctions of $\square$, and they form a complete set for the representation of any symmetric rank-two tensor. What this means is that any such tensor, $Q_{a b}$, can be represented as a sum of the form

$$
Q_{a b}=\sum_{0}^{\infty} \alpha_{n} n_{a b}^{n}+\sum_{1}^{\infty} \beta_{n} v_{a b}^{n}+\sum_{2}^{\infty} \gamma_{n} w_{a b}^{n}+\sum_{0}^{\infty} \delta_{n} \chi_{a b}^{n}
$$


for a unique set of constant coefficients $\left\{\alpha_{n}, \beta_{n}, \gamma_{n}, \delta_{n}\right\}$. If the eigenfunctions are appropriately normalized, so that

$$
\begin{aligned}
\delta_{n m} & =\int h_{a b}^{n}(x) h_{m}^{a b}(x) d v=\ldots=\int X_{a b}^{n}(x) X_{m}^{a b}(x) d V, \\
0 & =\int h_{a b}^{n}(x) v_{m}^{a b}(x) d v=\ldots=\int w_{a b}^{n}(x) X_{m}^{a b}(x) d V,
\end{aligned}
$$

then it is easy to show from equations (2.5) and (2.6) that the delta function defined by (2.4) satisfies

$$
Q^{a^{\prime} b^{\prime}}\left(x^{\prime}\right)=\int Q^{a b}(x) \delta_{\left(a^{a}\right.}^{a^{\prime}}\left(x, x^{\prime}\right) \delta_{b}^{b^{\prime}}\left(x, x^{\prime}\right) d v_{X}
$$

for any symmetric tensor $Q_{a b}$.

The nice thing about this method is that we don't have to explicitly construct any of the eigenfunctions $h_{n}^{a b}, \ldots, x_{n}^{a b}$. We will only need basic information about their eigenvalues and multiplicity which can be found in many articles $|21|$ and which can be obtained entirely from the group representation theory of so(5). The basic facts are as follows. The details, including the normalizations and eigenvalues of these modes, can be found in reference $|20|$. The ten degrees of freedom in the symmetric tensor $Q_{a b}(2.5)$ are divided among the different modes. There are five degrees of freedom in the tensor modes $h_{n}^{a b}$, which are transverse and traceless (TT).

$$
\begin{gathered}
0=n_{n}^{a b} g_{a b}=\nabla_{a} h_{n}^{a b} \\
\square h_{n}^{a b}=\lambda_{n}^{(2)} h_{n}^{a b} \\
\lambda_{n}^{(2)}=-\frac{\Lambda}{3}\left(n^{2}+7 n+8\right)
\end{gathered}
$$

There are three degrees of freedom in the modes $v_{n}^{a b}$ which are the symmetrized derivatives of transverse vectors.

$$
\begin{aligned}
v_{n}^{a b} & \left.=\left[-\frac{1}{2}\left(\lambda_{n}^{(1)}+\Lambda\right)\right]^{-1 / 2} \nabla^{(a} \xi_{n}^{b}\right) \\
0 & =\nabla_{a} \xi_{n}^{a} \\
\square v_{n}^{a b} & =\left(\lambda_{n}^{(1)}+\frac{5}{3} \Lambda\right) v_{n}^{a b} \\
\lambda_{n}^{(1)} & =-\frac{\Lambda}{3}\left(n^{2}+5 n+3\right)
\end{aligned}
$$

There is one degree of freedom in the modes $w_{n}^{a b}$ which are the traceless derivatives of longitudinal vectors 


$$
\begin{aligned}
w_{n}^{a b} & =\left[\lambda_{n}^{(0)}\left(3 / 4 \lambda_{n}^{(0)}+\Lambda\right)\right]^{-1 / 2}\left(\nabla^{a} \nabla^{b}-\frac{1}{4} g^{a b} \square\right) \phi_{n} \\
\square w_{n}^{a b} & =\left(\lambda_{n}^{(0)}+8 / 3 \Lambda\right) w_{n}^{a b} .
\end{aligned}
$$

Finally there is one degree of freedom in the pure-trace scalar modes $\chi_{n}^{a b}$.

$$
\begin{aligned}
\chi_{n}^{a b} & =1 / 2 g^{a b} \emptyset_{n} \\
\square X_{n}^{a b} & =\lambda_{n}^{(0)} X_{n}^{a b} \\
\lambda_{n}^{(0)} & =-\frac{\Lambda}{3} n(n+3)
\end{aligned}
$$

From a detailed examination of these modes and their normalizations (for which see $|20|)$ it can be shown that

$$
\nabla_{a} \nabla_{b} w_{n}^{a b}=\lambda_{n}^{(0)}\left(3 / 4 \lambda_{n}^{(0)}+\Lambda\right) \phi_{n}
$$

$\left(g_{a b} \nabla_{c} \nabla_{d}+g_{c d} \nabla_{a} \nabla_{b}\right) x_{n}^{c d}=2\left[\lambda_{n}^{(0)}\left(3 / 4 \lambda_{n}^{(0)}+\Lambda\right)\right]^{\frac{1}{2}} w_{a b}^{n}+2 \lambda_{n}^{(0)} x_{a b}^{n}$.

This is all that we will need to know about the mode functions.

As we said earlier, the path integral calculation of the propagator $|20|$ justifies our use of the following ansatz for the propagator:

$$
\begin{aligned}
& G^{a b a^{\prime} b^{\prime}}\left(x, x^{\prime}\right)=\sum_{0}^{\infty}\left\{\begin{array}{l}
\alpha_{n} h_{n}^{a b}(x) h_{n}^{a^{\prime} b^{\prime}}\left(x^{\prime}\right)+ \\
\beta_{n} v_{n}^{a b}(x) v_{n}^{a^{\prime} b^{\prime}}\left(x^{\prime}\right)+
\end{array}\right. \\
& \gamma_{n} w_{n}^{a b}(x) w_{n}^{a^{\prime} b^{\prime}}\left(x^{\prime}\right)+ \\
& \delta_{n} x_{n}^{a b}(x) x_{n}^{a^{\prime} b^{\prime}}\left(x^{\prime}\right)+ \\
& \left.\sigma_{n}\left[x_{n}^{a b}(x) w_{n}^{a^{\prime} b^{\prime}}\left(x^{\prime}\right)+w_{n}^{a b}(x) x_{n}^{a^{\prime} b^{\prime}}\left(x^{\prime}\right)\right]\right\} \text {. }
\end{aligned}
$$

Applying the wave operator $w_{\text {abcd }}$ to the propagator (2.13) and demanding that it yield the delta function (2.4) gives linear equations that uniquely determine the coefficients $\alpha_{n}, \ldots, \sigma_{n}$ to be

$$
\begin{array}{ll}
\alpha_{n}=k\left(-\lambda_{n}^{(2)}+2 / 3 \Lambda\right)^{-1} & \text { for } n>0 \\
\beta_{n}=k\left(-\lambda_{n}^{(1)}-\Lambda\right)^{-1} & \text { for } n \geqslant 1, \text { else zero. } \\
\gamma_{n}=k\left(-\lambda_{n}^{(0)}(1-\varepsilon)+\Lambda\right)^{-2}\left[\left(2 \varepsilon^{2}-\varepsilon-1 / 4\right) \lambda_{n}^{(0)}-\Lambda / 2\right] \text { for } n>2, \text { else zero. }
\end{array}
$$




$$
\begin{aligned}
& \delta_{n}=k\left(1 / 4 \lambda_{n}^{(0)}+\Lambda_{/ 2}\right)\left(\lambda_{n}^{(0)}(1-\varepsilon)+\Lambda\right)^{-2} \text { for } n \geqslant 0 \\
& \sigma_{n}=k\left(\lambda_{n}^{(0)}(1-\varepsilon)+\Lambda\right)^{-2}(\varepsilon-1 / 2)\left(\lambda_{n}^{(0)}\left(3 / 4 \lambda_{n}^{(0)}+\Lambda\right)\right)^{\frac{1}{2}} \text { for } n \geqslant 2, \text { else zero. }
\end{aligned}
$$

Here the constant $K=64 \pi G$ where $G$ is Newton's constant. With the propagator now determined by (2.13) and (2.14), we can discuss its infra-red behavior.

\section{Infra-red Behavior of the Graviton Propagator.}

We begin the discussion of the infra-red behavior of the graviton propagator by asserting that the propagator is finite for separated points if and only if all of the coefficients $\alpha_{n}, \ldots, \sigma_{n}$ are finite. This is indeed the case, provided that the gauge-fixing parameter $\mathcal{E}$ does not have one of the values

$$
\varepsilon_{\text {exceptional }}=1+\Lambda / \lambda_{n}^{(0)}=\frac{n^{2}+3 n-3}{n(n+3)}
$$

for $n=1,2, \ldots, \infty$. Now let us prove our assertion.

The first terms in the mode sum for the propagator, corresponding to $\alpha_{n}{ }_{n} h_{n}$ and $\beta_{n} V_{n} V_{n}^{\prime}$, have been evaluated by Allen and Turyn $|22|$ and shown to be completely finite. This leaves the final three terms, which can be related to the scalar propagator, for different values of the scalar mass. Thus to understand the infra-red behavior of the graviton propagator, all we have to do is understand the scalar case.

Here the situation is very simple. For two points $X$ and $X^{\prime}$, separated by a geodesic distance $\mu\left(x, X^{\prime}\right)$, the massive scalar propagator is $|18,20,22|$,

$G\left(m^{2}, \mu\right)=\sum_{0}^{\infty} \frac{\varphi_{n}(x) \varphi_{n}\left(X^{\prime}\right)}{-\lambda_{n}^{(0)}+m^{2}}=\frac{\Gamma(3 / 2+v) \Gamma(3 / 2-V)}{16 \pi^{2} a^{2}} F\left(3 / 2+V, 3 / 2-V ; 2 ; \cos ^{2}(\mu / 2 a)\right)$.

The right hand side of this equation, and hence the mode sum, is completely finite provided that $3 / 2-V$ is a not a nonpositive integer. Since $V=\left(9 / 4-a^{2} m^{2}\right)^{\frac{1}{2}}$, this means the propagator is finite provided that $\mathrm{m}^{2}$ does not take one of the (negative) values

$$
m^{2}=-\frac{1}{a^{2}} n(n+3) \quad \text { for } n=0,1,2, \ldots
$$

But these are exactly the values of $m^{2}$ for which the denominator $-\lambda_{n}^{(0)}+m^{2}$ in the mode sum vanishes! Exactly the same analysis applies to the "scalar" parts of the graviton propagator. We have thus proved that provided that if $\varepsilon$ is not given one of the "exceptional" values given above (3.1), the propagator is completely finite. What we will now do is to show why this is. 


\section{How Can The Gauge-Fixing Term Fail?}

The infra-red divergence that occurs in the propagator for the exceptional values of $\mathcal{E}$ can be easily understood. Imagine expressing the propagator as a path integral, or average, over all field configurations. If the gauge-fixing term was not present, then this integral would yield infinity, because it would include an infinite number of gauge-equivalent field configurations which had the same value of the action. The purpose of the gauge fixing term is to make the integral converge by giving gaugeequivalent field configurations different values of the action. Thus the gauge-fixing term "fails to do its duty" if there exist a distinct pair of configurations which are physically gauge equivalent and which have the same value of the gauge-fixed action. Let us now show that this is exactly what happens if $\varepsilon$ is given one of the "exceptional" values.

We can write the gauge fixing term (1.5) in the following form, after integrating by parts.

$$
S_{\text {gauge }}=-\alpha \int\left(h^{a b}-\varepsilon g^{a b} h_{e}^{e}\right) \nabla_{a} \nabla^{c}\left(h_{b c}-g_{b c} h_{d}^{d}\right) d\left(V_{0} l\right) .
$$

Now consider the following gauge transformation: $h^{a b} \rightarrow h^{a b}+\nabla^{(a b)}$ where $v^{b}=\nabla^{b} \varphi_{n}$ for the scalar mode $\varphi_{n}$, and $n \geqslant 1$. It is easy to verify that for $n>0, \nabla^{(a, b)}$ is nonzero, and

$$
S_{\text {gauge }}\left[\nabla^{\left(a_{v} b\right)}\right]=2 \alpha\left[\lambda_{n}^{(0)}(1-\varepsilon)+\Lambda\right]^{2}=2 \alpha \frac{\Lambda^{2}}{9} n^{2}(n+3)^{2}\left[\varepsilon-\frac{n^{2}+3 n-3}{n(n+3)}\right]^{2} .
$$

Thus, if $\mathcal{E}$ takes on one of the exceptional values - say the $n$ 'th exceptional valuethen the gauge-fixing term fails to be sensitive to the gauge transformation $h_{a b} \rightarrow h_{a b}+\nabla_{a} \nabla_{b} \varphi_{n}$ induced by the n'th scalar mode, because the r.h.s. vanishes! This is the source of the infra-red divergence that occurs for the exceptional values of $\mathcal{E}$. We will now show that this infra-red divergence, should it happen to arise because of a bad choice of $\boldsymbol{\varepsilon}$, is a harmless gauge artifact and makes no contribution to scattering amplitudes.

\section{The Infra-red Divergence is a Gauge-Artifact.}

Consider the tree level scattering process where two matter fields, which we denote $\varphi$, interact by exchanging a graviton. Here $\varphi$ could be any kind of matter, not just a scalar field. Schematically this looks like: 


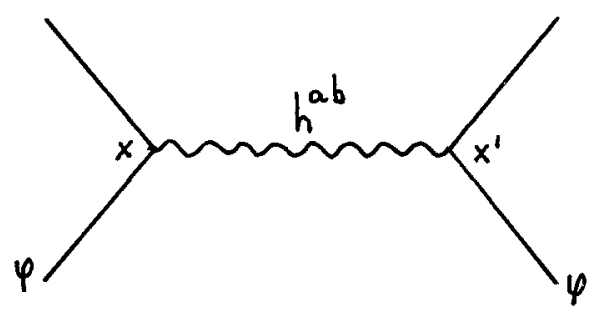

The amplitude for this process is determined by the stress tensor $T^{\text {ab }}$ of the matter. It is

$$
A=\iint T^{a b}(X) G_{a b c^{\prime} d^{\prime}}\left(X, X^{\prime}\right) T^{c^{\prime} d^{\prime}}\left(X^{\prime}\right) d V d V^{\prime},
$$

where $d V$ denotes the invariant four-volume element $\sqrt{g(x)} d^{4} x$ at the point $x$, and $d V^{\prime}$ denotes the same thing at $x^{\prime}$. Let us assume only that $\nabla_{a} T^{a b}=0$; ie that the operator $T^{a b}$, which is quadratic in the field $\varphi$, is conserved. This is true even in the presence of trace anomalies, for the renormalized operator, provided that it is a matrix element between physical (on-she11) states $|23|$. We will show that this amplitude is finite regardless of the value of the gauge-fixing parameter $\varepsilon$, and in particular for the "exceptional" values of $\varepsilon$, for which $G_{a b c} d^{\prime}$ contains infra-red divergences.

The amplitudes $A$ is a sum of five terms arising from the propagator (2.13). The first two terms are independent of $\varepsilon$. The final three terms, upon integration by parts, can be expressed as

$$
A_{\gamma}+A_{\delta}+A_{\sigma}=\iint T(x) \rho_{\varepsilon}\left(x, x^{\prime}\right) T\left(x^{\prime}\right) d V d V^{\prime}
$$

where $T(X)=T^{a}(X)$ is the trace of the stress tensor. The function $\rho_{\varepsilon}\left(X, X^{\prime}\right)$ is of the form

$$
\rho_{\varepsilon}\left(x, x^{\prime}\right)=c_{1}+c_{2}(\varepsilon-1 / 4)^{-2} \varphi_{1}(x) \varphi_{1}\left(x^{\prime}\right)+c_{3} \sum_{n=2}^{\infty} \frac{\varphi_{n}(x) \varphi_{n}\left(x^{\prime}\right)}{\lambda_{n}^{(0)}+\frac{4}{3} \Lambda} .
$$

Here $C_{1}, C_{2}$ and $c_{3}$ are nonzero constants. What matter is that there appears to be a single term in the amplitude that depends upon $\mathcal{E}$. However from gauge-invariance we known that the amplitude can not depend upon $\varepsilon$ at all ! We will now show that the second term above contributes nothing, even in the limit $\varepsilon \rightarrow 1 / 4$ !

The reason why is simple: the mode(s) $\varphi_{1}(x)$ obey $\nabla_{a} \nabla_{b} \varphi_{1}=-\frac{1}{3} \Lambda g_{a b} \varphi_{1}|24|$. Thus replacing $\mathrm{T}_{\mathrm{a}}^{\mathrm{a}} \varphi_{1}$ by $\mathrm{T}^{\mathrm{ab}} \nabla_{\mathrm{a}} \nabla_{\mathrm{b}} \varphi_{1}$, and integrating by parts $|25|$ to get $\left(\nabla_{a} \mathrm{~T}^{\mathrm{ab}}\right) \nabla_{\mathrm{b}} \varphi_{1}$, we see that the $\varepsilon$-dependent term vanishes as 1 ong as the stress tensor is conserved. What this means is that even in those cases where the two-point function has an infra-red divergence, the scattering amplitude is finite. This shows 
that in those cases where it occurs, infra-red divergence is a harmless gauge artifact.

\section{Conclusion.}

What has been shown in this talk is that the graviton propagator in de sitter space is 0K. If one makes a bad choice of gauge (-fixing term) then the propagator is infra-red divergent. However this is not a problem. You can either make a better choice of gauge (of which there are an infinite number), for which the propagator is completely finite, or else you can go right ahead and use the infra-red divergent one. We demonstrated that it doesn't matter. Gauge-invariance is the over-riding principle, and it ensures that even if the propagator has an infra-red divergence, the physical scattering amplitudes are finite.

A more detailed discussion of these points can also be found in an earlier published paper $|20|$. The complete closed form for the graviton propagator with $\mathcal{E}=1 / 2$ has also been found $|22|$. Finally a closed form in the de Sitter -non-invariant gauge (1.1) has been recently obtained $|26|$. This form applies to any spatially-flat Robertson-Walker mode1.

Acknowledgements.

I would like to thank S. Coleman, J. Iliopoulos and M. Turyn for helpful discussions.

\section{REFERENCES}

1 C.W. Misner, K.S. Thorne and J.A. Wheeler, Gravitation (Freedman, San Francisco, 1973) p. 410.

2 I. Antoniadis, J. Iliopoulos, T.N. Tomaras, Nucl.Phys. B261 (1985) 157.

I. Antoniadis and N.C. Tsamis, Phys.Lett. 144B (1984) 55.

E. Baum, Phys.Lett. 133B (1983) 185.

3 G. Gibbons, S.W. Hawking and S.T.C. Siklos, The Very Early Universe, Proceedings

of the Nuffield Workshop (Cambridge UP, 1983).

R. Brandenburger, Rev.Mod.Phys. 57 (1985) 1.

4 B. deWit and R. Gastmans, Nucl.Phys. B128 (1985) 1.

5 N.P. Myhrvold, Phys.Lett. 132B (1983) 308.

N.P. Myhrvold, Phys. Rev. D28 (1983) 2439.

E. Mottola, Phys.Rev. D31 (1985) 754.

E. Mottola, Phys.Rev. D33 (1986) 1616.

E. Mottola, NSF-ITP 85-33 preprint UCSB.

E. Mottola and P. Mazur, NSF-ITP 85-153 preprint UCSB.

S. Wada and T. Azuma, Phys.Lett. 132B (1983) 313.

6 P. Anderson, University of Florida at Gainesville preprint, 1985.

7 Gary T. Horowitz, Phys.Rev. D21 (1980) 1445.

8 G.W. Gibbons and S.W. Hawking, Phys.Rev. D15 (1977) 2738.

9 B. Allen, Ann.Phys. 161 (1985) 152.

1 B. Allen, Nucl.Phys. B226 (1983) 228.

10 S.W. Hawking and G.F.R. Ellis, The Large Scale Structure of Spacetime (Cambridge UP, 1980).

110 . Nachtmann, Commun. Math.Phys. 6 (1967) 1.

N.A. Chernikov and E.A. Tagirov, Ann. Inst. Henri Poincaré IX (1968) 109. 
J. Géhéniau and Ch. Schomblond, Bull.Cl.Sci., V.Ser.Acad.R.Belg. 54 (1968) 1147.

E.A. Tagirov, Ann. Phys. 76 (1973) 561.

P. Candelas and D.J. Raine, Phys.Rev. D12 (1975) 965.

Ch. Schomblond and P. Spindel, Ann. Inst. Henri Poincaré XXV (1976) 67.

T.S. Bunch and P.C.W. Davies, Proc.Roy. Soc.Lond. A360 (1978) 117.

B. Allen, Phys.Rev. D32 (1985) 3136.

B. Allen and T. Jacobson, Commun. Math.Phys. 103 (1986) 669.

B. Allen and C.A. Lütken, Commun. Math.Phys. 106 (1986) 201.

120 . Nachtman in reference 11 .

0 . Nachtman, Z. Phys. 208 (1968) 113.

0. Nachtman, Sitzungsber. Oesterr.Akad.Wiss.Math.Naturwiss.KI. 167 (1968) 363.

G.W. Gibbons and M.J. Perry, Proc.R.Soc.Lond. A358 (1978) 467.

13 I. Antoniadis, J. Iliopoulos and T.N. Tomaras, Phys.Rev.Lett. 56 (1986) 1319.

14 C. Itzykson and J.B. Zuber, Quantum Field Theory (McGraw-Hi11, NY, 1980).

15 S. Coleman and E.J. Weinberg, Phys. Rev. D7 (1973) 1888.

16 This of course is the infinitesimal form of the gauge transformation.

To generate finite transformations we have to go to higher order in $V$.

17 The Fadeev-Popov determinant $\left|h^{\mathrm{ab}}\right|$ does not depend upon $h^{\mathrm{ab}}$ at one-ioop, and thus does not contribute to the tree-level propagator. We have therefore left this Jacobian out of the formula for Gaba'b'.

18 B. Allen and T. Jacobson in reference 11 .

19 S.M. Christensen and M.J. Duff, Nucl.Phys. B170 (1980) 480.

N.H. Barth and S.M. Christensen, Phys.Rev. D28 (1983) 1876.

B. Allen, Phys. Rev. D34 (1986) 3670 .

$20 \mathrm{~B}$. Allen in reference 19.

21 S.L. Adler, Phys.Rev. D6 (1972) 3445, D8 (1973) 2400.

R. Raczka, N. Limic and J. Nierderle, J.Math.Phys. 7 (1966) 1861, 7 (1966) 2026, 8 (1967) 1079.

G.W. Gibbons and M.J. Perry, Nucl.Phys. B146 (1978) 90.

S.M. Christensen, M. Duff, G.W. Gibbons, and M.J. Perry, Phys.Rev.Lett. 45 (1980)161.

A. Higuchi, Yale Preprint YTP 85-22 (1985).

A. Chodos, E. Meyers, Ann.Phys. (NY) 156 (1984) 412.

M.A. Rubin and C.R. Ordonez, J.Math.Phys. 25 (1984) 2888, 26 (1985) 65.

22 B. Allen and $M$. Turyn, The graviton propagator in maximally symmetric spacer, Tufts University preprint (1986).

23 R. Wald, Phys. Rev. D17 (1978) 1477.

R. Wald, Commun. Math.Phys. 54 (1977) 1.

S.A. Fulling, M. Sweeny and R. Wald, Commun. Math.Phys. 63 (1978) 259.

24 In fact the mode that we have labeled $\phi_{1}$ is degenerate. There are five such modes with the same eigenvalue. If the four-sphere is $x^{2}+\ldots+x_{5}^{2}=1$ then the modes $\phi j$ are proportional to the $i^{\prime}$ th coordinate $x_{j}$.

25 The boundary terms can be shown to vanish in the Lorentzian spacetime case -see reference 20.

$26 \mathrm{~B}$. Allen, The graviton propagator in homogeneous and isotropic spacetimes, Tufts University Preprint TUTP 86-14 (1986).

(submitted to Nucl. Phys.) 